\title{
Blood Bank Service: An Experience at Newly Established Birat Medical College and Teaching Hospital, Tankisinuwari, Morang, Nepal
}

\author{
Kafle SU, ${ }^{1}$ Jha KK, ${ }^{2}$ Sigh $M,{ }^{2}$ Rana $R^{2}$
}

\section{Affiliation}

1. Associate Professor, Department of Pathology, Birat Medical College and Teaching Hospital, Tankisinuwari, Morang, Nepal

2 Lecturer, Department of Pathology, Birat Medical College and Teaching Hospital, Tankisinuwari, Morang, Nepal

\section{ARTICLE INFO}

Article History

Received : 2 April 2017

Accepted : 28 April 2017

Published : 30 April 2017

(C) Authors retain copyright and grant the journal right of first publication with the work simultaneously licensed under Creative Commons Attribution License CC - BY 4.0 that allows others to share the work with an acknowledgment of the work's authorship and initial publication in this journal.

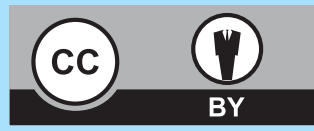

\section{* Corresponding Author}

Dr. Santosh Upadhyaya Kafle Associate Professor

Department of Pathology

Birat Medical College and Teaching Hospital

Tankisinuwari, Morang, Nepal

Email: drsantoshkafle@gmail.com

\section{Citation}

Kafle SU, Jha KK, Sigh M, Rana R. Blood Bank Service: An Experience at Newly Established Birat Medical College and Teaching Hospital Tankisinuwari, Morang, Nepal. BJHS 2017; 1 (1) 1:98-101

\section{ABSTRACT}

\section{Introduction}

Blood bank service facility of blood collection, storage and dispatching began from 19 June 2016 in the new blood bank and transfusion service unit, at Birat Medical College \& Teaching Hospital, Tankisinuwari, Morang, Nepal, with the support of Nepal Red Cross Society. As the supply of blood is finite, the inappropriate use of blood transfusion units and its component in the world exist, hence there is a need to monitor and regulate these services.

\section{Objectives}

The objective of this study is to know and identify different blood groups and its utilization pattern to meet the demand for future preparedness and to identify the total blood unit, which are being, replaced back.

\section{Methodology}

This retrospective study was conducted at BMCTH, Tankisinuwari, Morang for component dispatches from 19th June, 2016 to19th February, 2017. Requisitions for whole blood requirement for patients from various departments were reviewed regarding the department request, the whole blood requested and whether being replaced, blood group and socio-demographic profile.

\section{Results}

Out of 193 transfusion units dispatched, 28 patients were male and 165 patients were females respectively $B$ positive blood groups were most common, followed by $A$ positive, $O$ positive, $A B$ positive, both $A B$ and $O$ negative respectively. The largest numbers of requisitions were from Gynecology and Obstetrics ward in the hospital, while 22 requisites were from other health centers. Majority of the requisitions were for $51-60$ years age group from the local district Morang. The total number of blood units replaced were 38, out of which 5 for males and 33 for females respectively.

\section{Conclusion}

B positive was the predominant requested blood group, with Gynecology and Obstetrics ward demanding the most requests, among 51-60 years age group from Morang district. The total blood units being replaced were very less in number with some requisition forms, which lacked essential details.

\section{KEY WORDS}

Blood bank, blood group, B positive, transfusion unit 


\section{INTRODUCTION}

The blood transfusion units and the individual blood components have become an integral part of patient management in modern medicine. It can be vital and life saving procedures when given appropriately. In Nepal, the Nepal Red Cross Society (NRCS) began to provide institution based Blood Transfusion Service (BTS) through the Department of Pathology at Bir Hospital (1964). The first BTS was established as Laxmi Blood Bank in 1966, with the purpose to ensure an adequate, safe and timely supply of blood and blood products to meet the transfusion requirements of the people of $\mathrm{Nepal}$ in an equitable and affordable manner.

Blood bank service facility of blood collection, storage and dispatch began from 19 June 2016 in the new blood bank and transfusion service unit, at Birat Medical College and Teaching Hospital, Tankisinuwari, Morang, Nepal. It is established under the supervision of Nepal Red Cross Society of Eastern Region, Nepal. Like drugs, the blood and its component have property, which may cause the adverse reactions in the recipients, though they are important part of the patient management treatment protocols. ${ }^{1}$ Therefore, the physicians should be alert regarding the proper use and indication for requesting the blood transfusion units and its components. Further of which, the unwanted and unnecessary exposure and misuse of the blood transfusion recipient to numerous infectious as well as other noninfectious complications is prevented. So, the continual and regular auditing on proper usage of blood transfusion units and its components is mandatory. This in long term would help to track down the appropriate blood transfusion units and its component of utilization pattern and to set up the perfect guidelines in all the blood using specialties. Among such, the exact updates and proper auditing proves to be a useful management application for the justification of appropriateness and efficacy of blood and its component transfusion therapy. ${ }^{2}$ Not only this, but this could be a key factor of the quality assurance programme providing the necessary information for improving the blood transfusion medicine practice. ${ }^{3}$ In view of high rate of inappropriate use of blood and its component services around the world, there is a need to monitor and regulate these services. The objective of our study is to identify the mode of blood utilization pattern to meet the demand for future preparedness. And also to identify those blood units, which are being replaced.

\section{METHODOLOGY}

The study is retrospective and conducted at Department of Pathology, Birat Medical College and Teaching Hospital, Tankisinuwari, Morang, Nepal. For this study, all the required information and data from 19 June 2016 to 19 February 2017 were retrieved from the main registration record book of the Blood Bank Service Unit of Nepal Red Cross Society (NRCS) at Birat Medical College and Teaching Hospital. Among these, the requisitions for requiring whole blood for the patients from various departments were reviewed regarding the department request, the number of whole blood requested and whether the blood was replaced back, blood group and socio-demographic profile (age and gender) for the above period. Descriptive statistical analysis was done.

\section{RESULTS}

The present study was conducted for 193 transfusion blood units dispatched for different various blood groups over a period of 8 months from 19 June 2016 to 19 February 2017. Out of total 193 transfusion units, 28(14.51\%) patients were male and 165 (85.49\%) were females (Figure 1). Out of the blood groups units being dispatched, B positive were most common of 74 (38.35\%) followed by A positive of 55 (28.50\%), O positive of 49 (25.38\%), AB positive of 13 (6.74\%) and of $A B$ negative and $O$ negative as 1 each respectively (Table 1). The largest numbers of requisitions for 95 transfusion units were from Gynecology and Obstetrics ward, followed by General Surgery, Internal Medicine and ENT ward for 30, 11 and 2 transfusion units respectively (Table 2). Fifty five requisites were from other hospital outside the Birat Medical College and Teaching Hospital. Majority of the requisitions were for 51- 60 years age group from the local district Morang followed by neighboring district Sunsari. The total blood units replaced were 38 , out of which 5 units were from males and 33 units were from females.

Figure 1: Sex distribution $(n=193)$

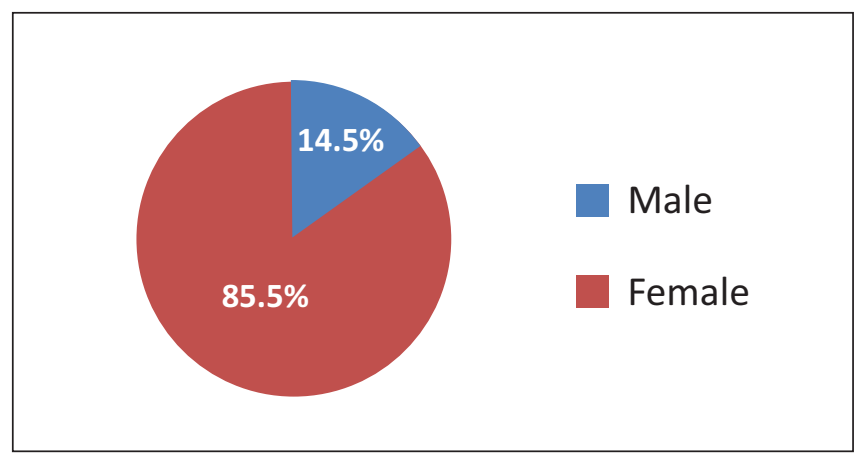

Table 1: Blood group distribution $(n=193)$

\begin{tabular}{ll} 
Blood Group & N $(\%)$ \\
\hline B Positive & $74(38.35 \%)$ \\
\hline A Positive & $55(28.50 \%)$ \\
\hline O Positive & $49(25.38 \%)$ \\
\hline AB Positive & $13(06.74 \%)$ \\
\hline AB Negative & $01(0.51 \%)$ \\
\hline O Negative & $01(0.51 \%)$ \\
\hline
\end{tabular}


Table 2: Requests from Departments ( $n=193$ )

\section{Department}

Gynecology\& Obstetrics

Surgery

Internal Medicine

ENT

Other health centers
Number

95

30

11

02

55

\section{DISCUSSIONS}

This retrospective study describes the different patterns of blood utilization along with the preparation in future for the demand of different blood groups in the Birat Medical College, Tankisinuwari, Nepal along with other nearby and surrounding health centers. A study conducted by Venkatachalapathy and $\mathrm{Das}^{4}$ concluded the importance of periodic review of the blood and its component usage pattern in hospital. According to the study, introducing, implementing and establishing the appropriate plans and policies guidelines for use of various blood transfusion units and blood components definitely help to decline the improper use of blood units and its components. Not only this, but instead it will ensure the availability of the blood transfusion units and its component to other patients who has emergency requirement of such components. Also, many patients suffering from unwanted blood transfusion related reactions can be saved as well. Likewise, our study also demonstrates the very initial status and figures of the newly established blood storage, collection and dispatch centers. In addition, being confined to the tertiary care medical college and teaching hospital with almost all existing departments of medical education, the future demand and use of such blood and its components would increase in number. The result of our study revealed more numbers of female than male patients from Morang district followed by Sunsari district demanding the whole blood. This has definitely linked to the higher numbers of patients demanding the blood were from Obstetrics and Gynecology departments followed by the General surgery and other wards respectively in ours hospital and nearby geographical areas. Among which, the maximum age group of the patients were fifth and sixth decade of life. The recorded data showed that 55 requisites of blood units were from the patients belonging to other health centers besides Birat Medical College and Teaching Hospital. This ultimately becomes a useful tool in making the different blood transfusion related strategy and policies for future preparedness and action. In another way, we can also predict from the result about the vulnerable age group, geographical areas and the type of diseases having the exact indication of blood transfusion.

Similarly, our study concluded that majority of blood group dispatched was $B$ positive out of total 193 blood units being dispatched followed by $A$ positive, $O$ positive, $A B$ positive, $O$ negative and $A B$ negative respectively. This sort of the internal audit data not only helps in tracing out the exact use and preparedness for future demand of the individual blood units, but also can play a vital function for quality control mechanism. These facts in detail have been prescribed and proved in the studies done by McCullough J and Steeper TA et al and Silver $\mathrm{H}$ and Tahhan HR et al. ${ }^{5,6}$ According to them, the regular data updates and working upon it of the individual blood bank is proved to be an important aspect of the quality control mechanism in any blood bank, which is same as that in any other institutions or organizations. Studies done by Brandia $\mathrm{K}$ et al and Joshi GP et $\mathrm{al}^{7,8}$ has the common conclusion, stating about the easy and appropriate use of blood transfusion units and the blood components is on a high demand for its easy availability of sophisticated blood banking services. Thus, the blood bank should be regular enough for updating and fulfilling the requisites of this vital and life saving products. Moreover, it should be able at first to access, track, evaluate and even change if necessary the existing plans policies and systems of demanding the blood transfusion units or the blood components.

Considering these facts of the blood and its component being one of the life saving product, the blood bank should always try to maintain the replacing blood units after delivery or dispatching of each blood units. So that the blood units storing in the blood bank is always maintained for each and every demand of it. Similarly, our study showed the result of minimal number of blood units being replaced after dispatching of each blood units. Out of 193 total dispatched blood units, 33 female replaced only 38 blood units and male replaced 5 . Such tendency of replacing the dispatched blood units in the blood bank should be increased, so that every demand and requisites for blood units would be fulfilled to overcome any life threatening situations demanding the use of blood and its components. Likewise, our study showed that, some of the requisition forms demanding such blood units even lacked the necessary and important details. This was one of the unwanted findings we came across our study. In another way, we can interpret that the necessary details about the patients identification regarding the full name, age, sex, proper address, blood group (if already known), total blood units required, clinical or working diagnosis if possible etc. should be clearly mentioned in every such blood units requisition form. 
Finally, we might achieve more favorable result if up gradation of blood storage and other facilities are considered at the blood bank which requires dealing with more inventories. A software based system for monitoring stock and predicting the utilization is mandatory. Generating a list of donors who can be used at the time when blood shortage and to avoid unavailability would be a great step for regular supply of such blood units. In addition, regular education programs related to such matter of improving the performance of any related staffs would be a positive step towards increasing the trend of using the blood transfusion units and the individual blood components appropriately.

\section{CONCLUSIONS}

$B$ positive blood unit was the predominant requested blood units by Gynecology and Obstetrics ward at this institute making the most of requests. The total replaced blood units

\section{REFERENCES}

1. Vishwanathan C, Jain R, Kamath M. Blood utilization review in a tertiary care hospital. Indian J Haematol Blood Transf 1999; 17:26-31.

2. Luk C, Eckert KM, Barr RM, Chin-Yee IH. Prospective audit of the use of fresh-frozen plasma, based on Canadian Medical Association transfusion guidelines. CMAJ 2002; 166: 1539-1540.

3. Metz J, McGrath KM, Copperchini ML, Haeusler M, Haysom HE, et al. Appropriateness of transfusions of red cells, platelets and fresh frozen plasma. An audit in a tertiary care teaching hospital. Med J Aust 1995; 162: 572-573, 576-7.

4. Venkatachalapathy TS, Das S. A Prospective Audit of Blood Transfusion Requests in RI Jalappa Hospital and Research Centre for Blood and Blood Components. J Blood Lymph 2012; 2:106. doi:10.4172/21657831.1000106/ were very less in number. Some requisition forms lacked essential details. Finally, these results could be useful for estimating and planning the requirements for our newly established Blood Bank to meet the demand in future.

\section{LIMITATIONS OF STUDY}

Study was concluded with only data available from the registration record book maintained at the blood bank service at Birat Medical College and Teaching Hospital.

\section{ACKNOWLEDGMENT}

Authors would like to acknowledge all the staffs of the blood bank unit and Mr. Sanjit of Clinical Laboratory Service unit of Birat Medical College and Teaching Hospital.

\section{CONFLICT OF INTEREST}

None

5 Cullough J, Steeper TA, Connelly DP, Jackson B, Huntington $S$, et al. Platelet utilization in a university hospital. JAMA 1988; 259: 2414-2418.

6 Silver H, Tahhan HR, Anderson J, Lachman M A noncomputer-dependent prospective review of blood and blood component utilization. Transfusion 1992; 32: 260-265.

7 Brandis K, Richards B, Ghent A, Weinstein S. A strategy to reduce inappropriate red blood cell transfusion. Med J Aust 1994; 160: 721-722.

8 Joshi GP, Landers DF. Audit in transfusion practice. J Eval Clin Pract 1998; 4: 141-146. 